\title{
Berberine Alleviates Paclitaxel-Induced Neuropathy
}

\author{
Ramin Rezaee ${ }^{1,2,3,4}$, Alireza Monemi ${ }^{5}$, Mohammad Amin SadeghiBonjar ${ }^{6}$, Mahmoud \\ Hashemzaei ${ }^{6 *}$
}

${ }^{1}$ Clinical Research Unit, Faculty of Medicine, Mashhad University of Medical Sciences, Mashhad, Iran

${ }^{2}$ Neurogenic Inflammation Research Center, Mashhad University of Medical Sciences, Mashhad, Iran

${ }^{3}$ Aristotle University of Thessaloniki, Department of Chemical Engineering, Environmental Engineering Laboratory, University Campus, Thessaloniki 54124, Greece

${ }^{4}$ HERACLES Research Center on the Exposome and Health, Center for Interdisciplinary Research and Innovation, Balkan Center, Bldg.

$\mathrm{B}, 10$ th $\mathrm{km}$ Thessaloniki-Thermi, Greece

${ }^{5}$ Students research Committee, School of Medicine, North Khorasan University of Medical Sciences, Bojnurd, Iran

${ }^{6}$ Department of Pharmacodynamics and Toxicology, School of Pharmacy, Zabol University of Medical Sciences, Zabol, Iran

\section{Key Words}

berberine, paclitaxel, heat hyperalgesia, mice, neuropathic pain

\section{Abstract \\ Objectives: Paclitaxel (PTX) as an anticancer drug used against solid cancers, possesses adverse reactions such as neuropathic pain which has confined its use. PTX-induced neuropathic pain is mediated via activa- tion of oxidative stress. Berberine (BER), an isoquino- line phytochemical found in several plants, exerts strong antioxidant and painkilling properties. In the current study, we aimed to evaluate pain-relieving effect of BER in a mouse model of PTX-induced neuropathic pain.}

Methods: This study was done using 42 male albino mice that were randomly divided into 6 groups $(n=7)$ as follow: Sham-operated (not treated with PTX), negative control group (PTX-treated mice receiving normal saline), BER 5, 10, and $20 \mathrm{mg} / \mathrm{kg}$ (PTX-treated mice receiving BER) and positive control group (PTX-treated mice receiving imipramine $10 \mathrm{mg} / \mathrm{kg}$ ). Neuropathic pain was induced by intraperitoneal administration of four dos-

Received: Jan 10, 2018 Reviewed: Feb 11, 2019 Accepted: Mar 21, 2019

$(0$ This is an Open-Access article distributed under the terms of the Creative Common Attribution Non-Commercial License (http://creativecommons.org/licenses/by-nc/4.0/) which permits unrestricted noncommercial use, distribution, and reproduction in any medium, provided the original work is properly cited.

(2) This paper meets the requirements of KS X ISO 9706, ISO 9706-1994 and ANSI/NISO Z39.48-1992 (Permanence of Paper). es of PTX ( $2 \mathrm{mg} / \mathrm{kg} /$ day) on days $1,3,5$ and 7 . Then, on day 7 , hot plate test was done to assess latency to heat to measure possible anti-neuropathic pain effect of BER.

Results: Four doses of PTX $2 \mathrm{mg} / \mathrm{kg} /$ day induced neuropathy that was reduced by BER at all time-points (i.e. 0, 30, 60, 90 and $120 \mathrm{~min}$ ) after injection $(\mathrm{P}<0.001$ in comparison to control). The statistical analysis of data showed significant differences between groups ( $\mathrm{P}$ $<0.001$ in comparison to negative control), at 30, 60, 90 and $120 \mathrm{~min}$ after injection of BER 5, 10 and $20 \mathrm{mg} /$ $\mathrm{kg}$; in other words, 30, 60, 90 and 120 min after BER administration, neuropathic pain was significantly reduced as compared to normal saline-treated mice.

Conclusion: Altogether, our results showed that PTX could induce neuropathic pain as reflected by hyperalgesia and BER could alleviate PTX-induced thermal hyperalgesia.

\section{Introduction}

Peripheral neuropathy is considered one the main side effects of paclitaxel (PTX), an anti-cancer drug which is widely used to treat solid tumors (e.g. breast and ovarian cancer) $[1,2]$. This dose-limiting neurotoxicity is observed in the form of hypoesthesia, par-

"Corresponding Author

Mahmoud Hashemzaei. Department of Pharmacodynamics and Toxicology, School of Pharmacy, Zabol University of Medical Sciences, Zabol, Iran.

Tel: +98-91-5142-1037 FAX: +98-054-3223-2162

Email: mhtoxicologist@gmail.com 
esthesia, and distal extremities pain [3]. Moreover, higher doses of PTX are associated with hypoesthesia and anesthesia whereas lower doses induce hyperalgesia and allodynia [4-6]. Besides, following PTX administration, peripheral neuroinflammation as indicated by macrophage recruitment and increased cytokine/chemokine secretion $[7,8]$.

Berberine (BER) is a naturally occurring alkaloid (Figure 1) isolated from several plants such as Coptis chinensis, Coptis japonica, Berberis vulgaris and Berberis croatica [9]. It has been shown that BER has antiprotozoal, antimicrobial, and anti-inflammatory properties and traditionally, BER-containing plants have been considered a remedy for dysentery, diarrhea, stomatitis, and hepatitis [10-13]. For several decades, BER has been used in China as an OTC medicine to treat diarrhea [14]. Also, modern pharmacology has shown tumor-suppressing and apoptosis-inducing effects of BER, which make it a potential anti-cancer chemical $[15,16]$. The pharmacokinetic profile of BER shows that the oral bioavailability of this compound is very low ( $<1 \%$ ) [17]. Nevertheless, BER may have marked effects on the brain and CNS as it can cross blood-brain-barrier [18]. Furthermore, BER has exerted anti-inflammatory activities in various human and animal tissues such as the liver, adipose tissue, vascular endothelial cells, and intestine [19-22]. Mechanistically speaking, BER diminishes the expression of genes producing cytokines involved in inflammatory pathways (e.g. tumor necrosis factor- $\alpha$ (TNF- $\alpha$ ), interleukins, prostaglandins, cyclooxygenase-2 (COX-2), and inducible nitric oxide synthase (iNOS)) via interfering with AMP-activated protein kinase [23].

Considering PTX-induced peripheral neuropathy which is a major concern of its use, and based on the reports on the anti-inflammatory activities of BER, we were encour-<smiles>COc1ccc2cc3[n+](cc2c1OC)CCc1cc2c(cc1-3)OCO2</smiles>

Figure 1 Chemical structure of BER. aged to examine the effect of BER on PTX-induced neuropathy.

\section{Materials and Methods}

\subsection{Drugs and Chemicals}

Paclitaxel was obtained from Aboureihan Pharmaceutical Co (Iran) and BER chloride was purchased from Sigma-Aldrich (Germany).

\subsection{Animals and grouping}

This study was done in 42 male albino mice (30-35 g and 4 weeks old) obtained from Faculty of Pharmacy, Zabol University of Medical Sciences, Zabol, Iran. Animals were kept in Plexiglass cages $(n=7)$ at $22 \pm 2^{\circ} \mathrm{C}$ with $12 \mathrm{hr}: 12$ $\mathrm{hr}$ light/dark cycle and they had free access to food and water, ad libitum. Animals were randomly divided into 6 groups $(\mathrm{n}=7)$ as follows: Sham-operated group (mice that received intraperitoneal injections of normal saline instead of PTX), saline-treated group (PTX-treated mice that received normal saline and served as negative control) and BER groups (PTX-treated mice that received BER 5, 10, and $20 \mathrm{mg} / \mathrm{kg}$ ) as well as imipramine $10 \mathrm{mg} / \mathrm{kg}$ (PTX-treated mice that received imipramine and served as positive control). All animal experiments were done in accordance with the National Ethical Guidelines for use and care of laboratory animals.

\subsection{Administration of PTX and BER}

Based on previous studies [24, 25], a cumulative dose of $8 \mathrm{mg} / \mathrm{kg}$ was administered (PTX $2 \mathrm{mg} / \mathrm{kg}$ was administered on days 1, 3, 5 and 7) to mice. Also, a single dose of BER 5, 10, and $20 \mathrm{mg} / \mathrm{kg}$ was intraperitoneally injected on day 7. Doses and route chosen for BER administration were chosen based on previously published reports [26, 27].

\subsection{Hot-plate test}

On day 7, animals were placed on a hot-plate apparatus (Ugo Basile 35100, United Kingdom). In order to measure the pain, time to licking, lifting paws or jumping from the hot-plate surface (cut-off time was set at $45 \mathrm{sec}$ ) was regarded as the end-points for assessment of response to heat latency. Latencies were measured at 30, 60, 90 and 120 min after BER injection.

\subsection{Statistical Analysis}

One-way analysis of variance (ANOVA) was used for data analysis and Newman-Keuls test was used to check differences between negative control and other groups. A P < 0.05 was considered statistically significant. 
A

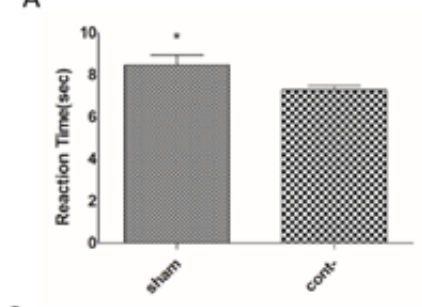

C
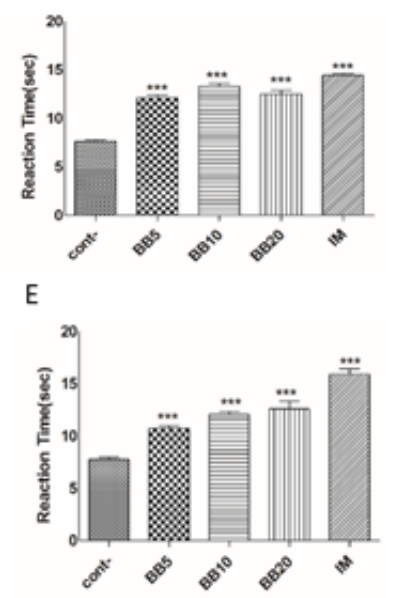

B

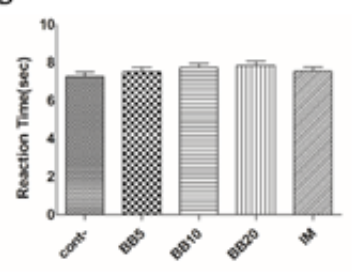

D
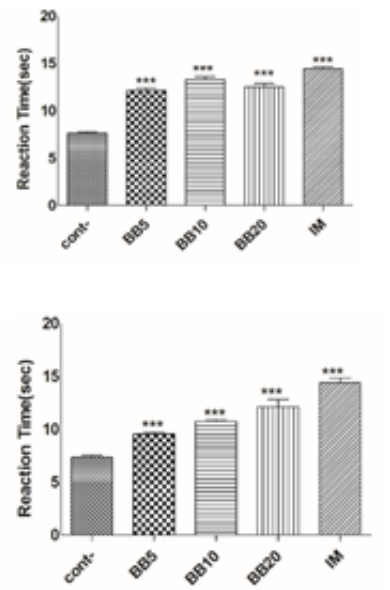

Figure 2 Effects of berberine (BER) on paclitaxel-induced neuropathic pain in mice. Latency to latency to heat was recorded at 0 (2B), 30 (2C), 60 (2D), 90 (2E) and 120(2F) min following treatment with B ER $\left({ }^{*} \mathrm{P}<0.05\right.$ and ${ }^{* * *} \mathrm{P}<0.001$ represent significant differences compared to the negative control). Cont: negative control; BB: Berberine; and IM: Imipramine.

\section{Results}

\subsection{Anti-neuropathic effects of BER meas- ured by hot plate test}

As shown in Figure 2A, administration of four doses of PTX $(2 \mathrm{mg} / \mathrm{kg} /$ day; i.p.) could induce hyperalgesia. As Figure 2B depicts, at time point 0 (i.e. 0 min after BER injection), there were no significant differences between control and BER-treated mice. At time-point 30, significant differences were observed between control and BER-treated groups (Figure 2C). Besides, differences were significant between control and BER 5 and $10 \mathrm{mg}$ at timepoint 60 (Figure 2D). At time-points 90 and 120 (Figures $2 \mathrm{E}$ and $2 \mathrm{~F}$, respectively) also significant differences were observed between control and treatment groups. Altogether, except for time-point 0 , at the other time-points, significant differences $(\mathrm{P}<0.001)$ were shown between negative control and BER-treated groups.

\section{Discussion}

PTX was originally isolated form the bark of pacific yew tree, Taxus brevifolia and is used to treat solid tumor via inducing cell mitosis arrest [28]. However, several adverse reactions such as myelosuppression, kidney damage and peripheral neurotoxicity have been reported for PTX [29]. Neuropathy which is characterized by hyperalgesia, allodynia, numbness, tingling, burning sensations confines PTX application and does not resolve even with cessation of PTX administration [29]. In spite of many studies on PTX-induced neuropathy, no cure has been proposed so far [29].

The results of the current study revealed that four doses of $2 \mathrm{mg} / \mathrm{kg} /$ day of PTX could induce neuropathic pain in mice and BER could significantly ameliorate it. Furthermore, a single dose of BER mitigated acute neuropathic pain produced by PTX in mice. BER is a benzylisoquinoline alkaloid found in theroot, rhizome and stem bark of numerous medicinal plants including Hydrastis canadensis, Coptis chinensis, Berberis aquifolium and Berberis vulgaris [30, 31]. BER has been investigated for its anti-hypertensive, antiarrhythmic, antihyperglycemic, anticancer, antidepressant, anxiolytic, neuroprotective, antioxidant, anti-inflammatory, analgesic and hyperlipidemic activities [3136]. Many clinical studies have well confirmed antioxidant effects of BER in diabetes, high cholesterol, and various inflammatory conditions such as Alzheimer's and cerebral ischemia [30, 33, 34].

PTX-induced neuropathy has been mostly attributed to ROS over-production and decrement of endogenous antioxidants [37]. As a natural alkaloid, BER shows antioxidant 
effects and it was found effective for treatment of diabetes, hyperlipidemia, and inflammation [26]. It has been cleared that inflammation and activation of mast cells are involved in neuropathic pain regardless of its cause [29] . BER is one of the strongest antioxidant that can reduce ROS production [31]. In a study, it was shown that quercetin, a polyphenolic flavonoid, could alleviate neuropathic pain by decreasing activation of mast cells. Also, many studies have confirmed anti-inflammatory effects of BER and showed that this effect might contribute to neuropathic pain- relieving properties of BER [38-40].

One of the most active oxidative component that is involved in neuropathy and is induced in diabetic animals, is 2,2-diphenyl-1-picrylhydrazyl (DPPH) [41]. In a study done by Shirwaikar et al, BER reduced DPPH levels in an animal model showing that BER anti-neuropathic effects are induced via different pathways [42].

Furthermore, BER can inhibit neurodegeneration via ROS, MAO (monoamine oxidases) and AchE (acetylcholinesterase) inhibition and GLP-1, Nrf2, Akt-PI3K, CREB and pCREB activation [43]. The aforementioned mechanisms have been investigated to develop new approaches for treatment of Alzheimer's, Parkinson's and other neurodegenerative diseases [43].

BER posed neuroprotective effects by activation of Akt$\mathrm{Pi} 3$ pathways in neuronal cell lines, decreased NF-kB inflammatory effects in neuronal cell lines and alleviated neuropathy in animal models [43]. Furthermore, shortterm administration of BER in acute stroke posed protective effects and this confirmed that beside the effect of long-term administration of BER, short-term usage of this compounds is also of considerable importance [43].

\section{Conclusion}

Altogether, our results showed that PTX could induce neuropathic pain as reflected by hyperalgesia and a single dose of BER could alleviate PTX-induced thermal hyperalgesia. As a limitation, the present study lacked mechanistic investigations, therefore, future studies should clarify the role of ROS-scavenging or antihistaminic effects of BER in its anti-neuropathic properties.

\section{Acknowledgment}

Authors would like to thank the Vice Chancellor of Research, Zabol University of Medical Sciences, Zabol, Iran.

\section{Conflict of interest}

The authors declare no conflict of interest.

\section{References}

1. Wilson L, Jordan M. New microtubule/tubulin-targeted anticancer drugs and novel chemotherapeutic strategies. Journal of chemotherapy; 2013.

2. Lee JJ, Swain SM. Peripheral neuropathy induced by microtubule-stabilizing agents. Journal of Clinical Oncology. 2006;24:1633-1642.
3. Park S, Krishnan A, Lin C, Goldstein D, Friedlander M, Kiernan M. Mechanisms underlying chemotherapy-induced neurotoxicity and the potential for neuroprotective strategies. Current medicinal chemistry. 2008;15:3081-3094.

4. Cliffer KD, Siuciak JA, Carson SR, Radley HE, Park JS, Lewis DR, et al. Physiological characterization of Taxol-induced large-fiber sensory neuropathy in the rat. Annals of neurology. 1998;43:46-55.

5. Polomano RC, Mannes AJ, Clark US, Bennett GJ. A painful peripheral neuropathy in the rat produced by the chemotherapeutic drug, paclitaxel. Pain. 2001;94:293304.

6. Flatters SJ, Bennett GJ. Studies of peripheral sensory nerves in paclitaxel-induced painful peripheral neuropathy: evidence for mitochondrial dysfunction. Pain. 2006;122:245-257.

7. Liu C-C, Lu N, Cui Y, Yang T, Zhao Z-Q, Xin W-J, et al. Prevention of paclitaxel-induced allodynia by minocycline: effect on loss of peripheral nerve fibers and infiltration of macrophages in rats. Molecular pain. 2010;6:76.

8. Naguib M, Xu JJ, Diaz P, Brown DL, Cogdell D, Bie B, et al. Prevention of paclitaxel-induced neuropathy through activation of the central cannabinoid type 2 receptor system. Anesthesia and analgesia. 2012;114:1104.

9. Kosalec I, Gregurek B, Kremer D, Zovko M, Sanković K, Karlović K. Croatian barberry (Berberis croatica Horvat): A new source of berberine-Analysis and antimicrobial activity. World Journal of Microbiology and Biotechnology. 2009;25(1):145-150.

10. Chang Y. Effectiveness of berberine in bacillary dysentery. Zhonghua nei ke za zhi. 1959;7:741-743.

11. Xia X, Wang H, Niu X, Liu Z, Liu Y, Qi Z, et al. Assessment of the anti-diarrhea function of compound Chinese herbal medicine cangpo oral liquid. African Journal of Traditional, Complementary and Alternative Medicines. 2013;11:140-147.

12. Jiang X-W, Zhang Y, Zhu Y-L, Zhang H, Lu K, Li F-F, et al. Effects of berberine gelatin on recurrent aphthous stomatitis: a randomized, placebo-controlled, double-blind trial in a Chinese cohort. Oral surgery, oral medicine, oral pathology and oral radiology. 2013;115:212-217.

13. Li HL, Han T, Liu RH, Zhang C, Chen HS, Zhang WD. Alkaloids from Corydalis saxicola and Their Anti-Hepatitis B Virus Activity. Chemistry \& biodiversity. 2008;5:777-783.

14. Yao J, Kong W, Jiang J. Learning from berberine: Treating chronic diseases through multiple targets. Science China Life Sciences. 2015;58:854-859.

15. Wu H-L, Chuang T-Y, Al-Hendy A, Diamond MP, Azziz $\mathrm{R}$, Chen Y-H. Berberine inhibits the proliferation of human uterine leiomyoma cells. Fertility and sterility. 2015;103:1098-1106.

16. Refaat A, Abdelhamed S, Saiki I, Sakurai H. Inhibition of p38 mitogen-activated protein kinase potentiates the apoptotic effect of berberine/tumor necrosis factor-related apoptosis-inducing ligand combination therapy. Oncology letters. 2015;10:1907-1911.

17. Liu Y-T, Hao H-P, Xie H-G, Lai L, Wang Q, Liu C-X, et 
al. Extensive intestinal first-pass elimination and predominant hepatic distribution of berberine explain its low plasma levels in rats. Drug metabolism and disposition. 2010;38:1779-1784.

18. Peng W-H, Wu C-R, Chen C-S, Chen C-F, Leu Z-C, Hsieh M-T. Anxiolytic effect of berberine on exploratory activity of the mouse in two experimental anxiety models: interaction with drugs acting at 5-HT receptors. Life Sciences. 2004;75:2451-2462.

19. Lou T, Zhang Z, Xi Z, Liu K, Li L, Liu B, et al. Berberine inhibits inflammatory response and ameliorates insulin resistance in hepatocytes. Inflammation. 2011;34:659-667.

20. Choi B, Ahn I, Kim Y, Park J, Lee S, Hyun C, et al. Berberine reduces the expression of adipogenic enzymes and inflammatory molecules of 3T3-L1 adipocyte. Experimental and Molecular Medicine. 2006;38:599.

21. Wu M, Wang J, Liu L-t. Advance of studies on anti-atherosclerosis mechanism of berberine. Chinese journal of integrative medicine. 2010;16:188-192.

22. Gu L, Li N, Gong J, Li Q, Zhu W, Li J. Berberine ameliorates intestinal epithelial tight-junction damage and down-regulates myosin light chain kinase pathways in a mouse model of endotoxinemia. Journal of Infectious Diseases. 2011;203:1602-1612.

23. Jeong HW, Hsu KC, Lee J-W, Ham M, Huh JY, Shin HJ, et al. Berberine suppresses proinflammatory responses through AMPK activation in macrophages. American Journal of Physiology-Endocrinology and Metabolism. 2009;296:E955-E964.

24. Toma W, Kyte SL, Bagdas D, Alkhlaif Y, Alsharari SD, Lichtman AH, et al. Effects of paclitaxel on the development of neuropathy and affective behaviors in the mouse. Neuropharmacology. 2017;117:305-315.

25. Segat GC, Manjavachi MN, Matias DO, Passos GF, Freitas CS, Costa R, et al. Antiallodynic effect of beta-caryophyllene on paclitaxel-induced peripheral neuropathy in mice. Neuropharmacology. 2017;125:207-219.

26. Kim SO, Kim HJ. Berberine ameliorates cold and mechanical allodynia in a rat model of diabetic neuropathy. Journal of medicinal food. 2013;16:511-517.

27. Mojarad TB, Roghani M. The anticonvulsant and antioxidant effects of berberine in kainate-induced temporal lobe epilepsy in rats. Basic and clinical neuroscience. 2014;5:124.

28. Wani MC, Taylor HL, Wall ME, Coggon P, McPhail AT. Plant antitumor agents. VI. Isolation and structure of taxol, a novel antileukemic and antitumor agent from Taxus brevifolia. Journal of the American Chemical Society. 1971;93:2325-2327.

29. Flatters SJ, Bennett GJ. Ethosuximide reverses paclitaxel-and vincristine-induced painful peripheral neuropathy. Pain. 2004;109:150-161.

30. Komal S, Ranjan B, Neelam C, Birendra S, Kumar SN. Berberis aristata: A review. Int J Res Ayurveda Pharm. 2011;2:383-388.

31. Kumar A, Chopra K, Mukherjee M, Pottabathini R, Dhull DK. Current knowledge and pharmacological profile of berberine: an update. European journal of pharmacology. 2015;761:288-297.

32. Liu C-S, Zheng Y-R, Zhang Y-F, Long X-Y. Research pro- gress on berberine with a special focus on its oral bioavailability. Fitoterapia. 2016;109:274-282.

33. Lan J, Zhao Y, Dong F, Yan Z, Zheng W, Fan J, et al. Meta-analysis of the effect and safety of berberine in the treatment of type 2 diabetes mellitus, hyperlipemia and hypertension. Journal of ethnopharmacology. 2015;161:69-81.

34. Wang N, Tan H-Y, Li L, Yuen M-F, Feng Y. Berberine and Coptidis Rhizoma as potential anticancer agents: Recent updates and future perspectives. Journal of ethnopharmacology. 2015;176:35-48.

35. Imenshahidi M, Qaredashi R, Hashemzaei M, Hosseinzadeh H. Inhibitory effect of berberis vulgaris aqueous extract on acquisition and reinstatement effects of morphine in conditioned place preferences (CPP) in mice. Jundishapur journal of natural pharmaceutical products. 2014;9(3):e16145.

36. Hassani FV, Hashemzaei M, Akbari E, Imenshahidi M, Hosseinzadeh $\mathrm{H}$. Effects of berberine on acquisition and reinstatement of morphine-induced conditioned place preference in mice. Avicenna journal of phytomedicine. 2016;6(2):198-204.

37. Alexandre J, Nicco C, Chéreau C, Laurent A, Weill B, Goldwasser F, et al. Improvement of the therapeutic index of anticancer drugs by the superoxide dismutase mimic mangafodipir. Journal of the National Cancer Institute. 2006;98:236-244.

38. Barbosa-Filho JM, Piuvezam MR, Moura MD, Silva MS, Lima KVB, da-Cunha EVL, et al. Anti-inflammatory activity of alkaloids: A twenty-century review. Revista Brasileira de Farmacognosia. 2006;16:109-139.

39. Sack RB, Froehlich JL. Berberine inhibits intestinal secretory response of Vibrio cholerae and Escherichia coli enterotoxins. Infection and immunity. 1982;35:471475.

40. Tsai C, Ochillo R. Pharmacological effects of berberine on the longitudinal muscle of the guinea-pig isolated ileum. Archives internationales de pharmacodynamie et de thérapie. 1990;310:116-131.

41. Kaeidi A, Esmaeili-Mahani S, Sheibani V, Abbasnejad M, Rasoulian B, Hajializadeh Z, et al. Olive (Olea europaea L.) leaf extract attenuates early diabetic neuropathic pain through prevention of high glucose-induced apoptosis: in vitro and in vivo studies. Journal of ethnopharmacology. 2011;136:188-196.

42. Shirwaikar A, Shirwaikar A, Rajendran K, Punitha ISR. In vitro antioxidant studies on the benzyl tetra isoquinoline alkaloid berberine. Biological and Pharmaceutical Bulletin. 2006;29:1906-1910.

43. Jiang W, Wei W, Gaertig MA, Li S, Li X-J. Therapeutic effect of berberine on Huntington's disease transgenic mouse model. PLoS One. 2015;10:e0134142. 